\section{TRIBAND BRANCH LINE COUPLER USING DOUBLE-LORENTZ TRANSMISSION LINES}

Hanseung Lee and Sangwook Nam

School of Electrical Engineering and INMC, Seoul National University, Seoul, Korea; Corresponding author: seung346@ael.snu.ac.kr

Received 21 September 2007

ABSTRACT: The concept of double-Lorentz (DL) transmission lines (TL) provides two additional degrees of freedom in realizing triband microwave devices. The triband operation is accomplished by the flexible phase slope characteristic of the DL TL with the frequency. In this article, the theory, design procedure, and implementation of the triband quarter-wave (N/4) DL TL are presented. Moreover, the triband N/4 open-circuit stub and triband branch-line coupler $(B L C)$ are introduced for the application of mobile TV service. The simulated and measured results of these triband components are also presented. The N/4 DL TL open-circuit stub demonstrates insertion loss larger than $17 \mathrm{~dB}$ at each target band. The triband BLC has S-parameters of output larger than $-4.569 d B$, return losses larger than $14 d B$, isolations larger than 15 $d B$, phase differences of output $90^{\circ} \pm 5^{\circ}$ at each target band. (C) 2008 Wiley Periodicals, Inc. Microwave Opt Technol Lett 50: 1174-1177, 2008; Published online in Wiley InterScience (www.interscience.wiley. com). DOI 10.1002/mop.23311

Key words: left-handed; double-Lorentz; transmission lines; triband; directional couplers

\section{INTRODUCTION}

The concept of artificial TLs having metamaterial properties in one-dimension has been a hot topic in a new field of science and technology [1-3]. Purely left-handed (PLH) are introduced by Eleftheriades in 2002. A CRLH transmission line is the expansion of the PLH because it includes a series inductance and a parallel capacitance, which always exist in the practical LC-network implementation of the PLH transmission line. As a dual concept of the conventional CRLH TL, the dual composite right/left-handed (D-CRLH) transmission line metamaterial was introduced and its properties were investigated by Caloz [4]. The practical artificial LC-network implementation of the D-CRLH transmission line

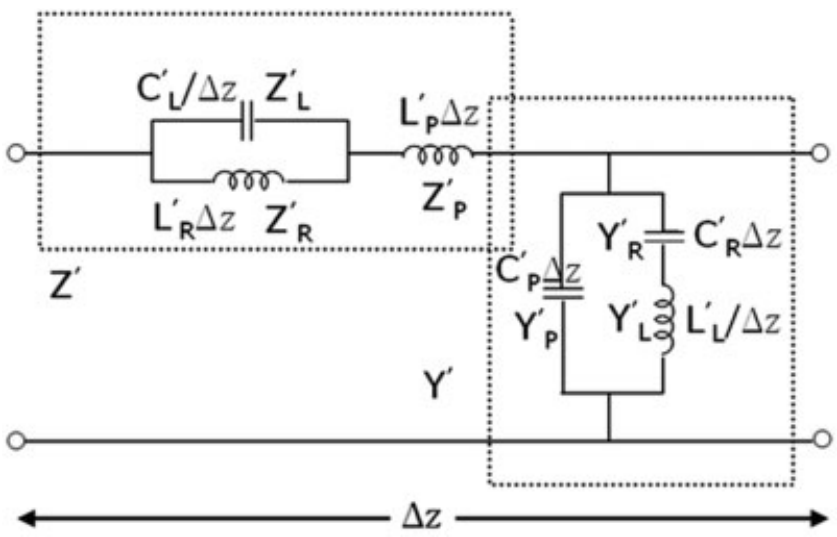

Figure 1 Equivalent circuit model for the Double-Lorentz transmission line. The subscripts R, L, and P stand for RH, LH, and "Parasitic," respectively, and the primes indicate per-unit-length RH and "Parasitic" or times-unit-length $\mathrm{LH}$ reactances, related to the per-unit length immitances $Z^{\prime}$ and $Y^{\prime}$. The incremental length $\Delta z$ is much smaller than the guided wavelength $\lambda_{\mathrm{g}}, \Delta z \lambda_{\mathrm{g}}$

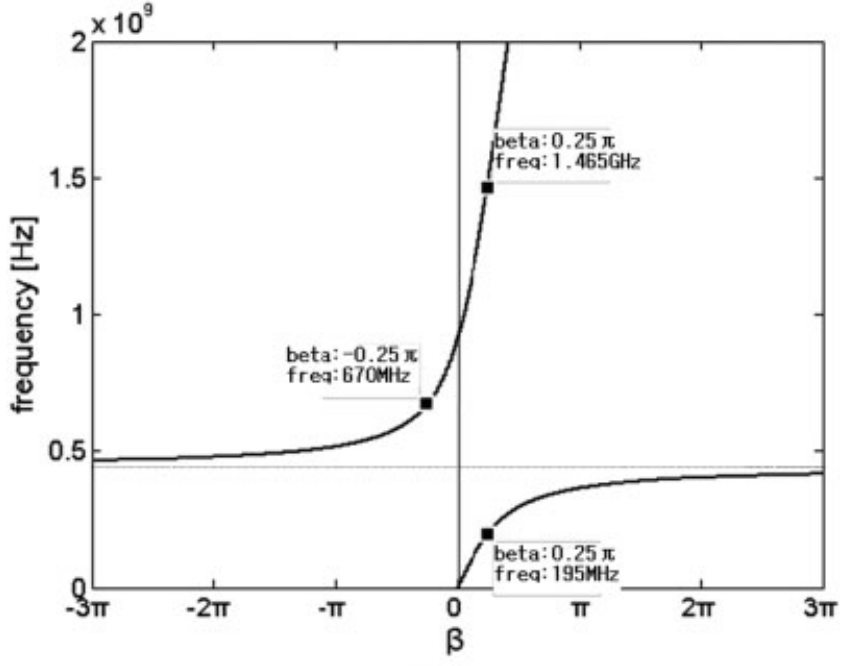

(a)

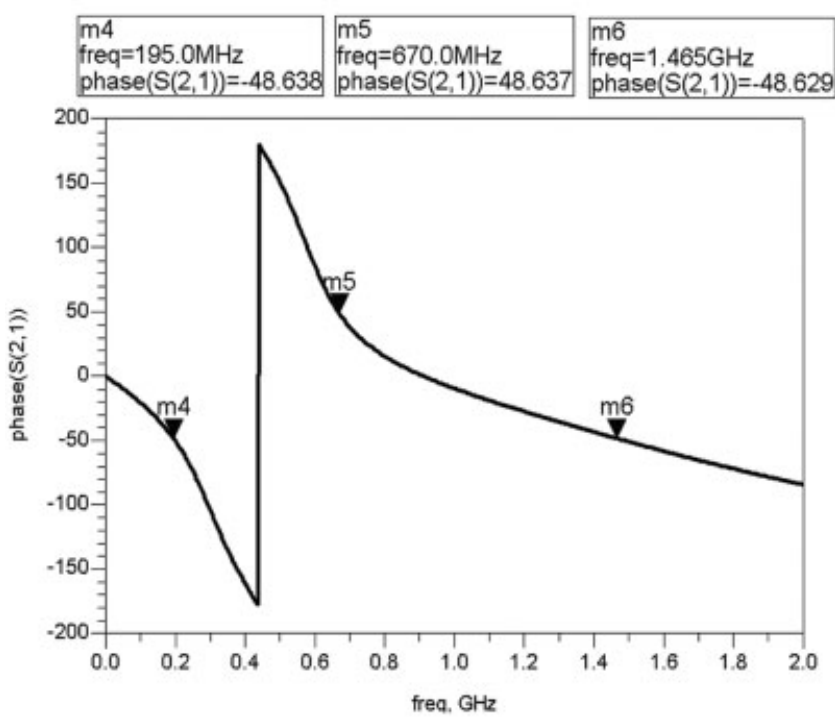

(b)

Figure 2 Triband property of the LC-network ( $N=1$ unit cell) DL with $\Delta z \rightarrow 0$. The parameters, $L_{\mathrm{R}}=20.68 \mathrm{nH}, C_{\mathrm{R}}=8.27 \mathrm{pF}, L_{\mathrm{L}}=35.75 \mathrm{nH}$, $C_{\mathrm{L}}=14.3 \mathrm{pF}, L_{\mathrm{P}}=5.134 \mathrm{nH}$, and $C_{\mathrm{P}}=2.053 \mathrm{pF}$ are designed to produce propagation constants of $\pi / 4,-\pi / 4$, and $\pi / 4$, respectively, at $195 \mathrm{MHz}$, $670 \mathrm{MHz}$, and $1.465 \mathrm{GHz}$. (a) Dispersion diagram computed by (1a). (b) $S$-parameters (phase), verifying the designed DL structure having the desired propagation constants at the target frequencies

always accompanies parasitic elements (a series inductance and a parallel capacitance) and these parasitic elements are regarded as important transmission line parameters; the novel transmission line metamaterial is referred as a double-Lorentz (DL) transmission line [5]. A DL TL metamaterial has an intrinsic triband property that can be used in triband microwave components. Triband components are salutary to reduce the size of devices used in recent multiband mobile communication systems. In wireless communication circuits, branch-line coupler (BLC) is used for dividing an input signal into two output signals with $90^{\circ}$ phase difference. The conventional BLC made by $\lambda / 4$ right-handed (RH) TL operates at a target frequency $f_{1}$ and at its odd harmonics [6]. However, wireless systems may demand arbitrary second or third operating frequencies, which are not a multiple of $f_{1}$. The BLC using DL TLs can be a solution of such problems because of its operation at 


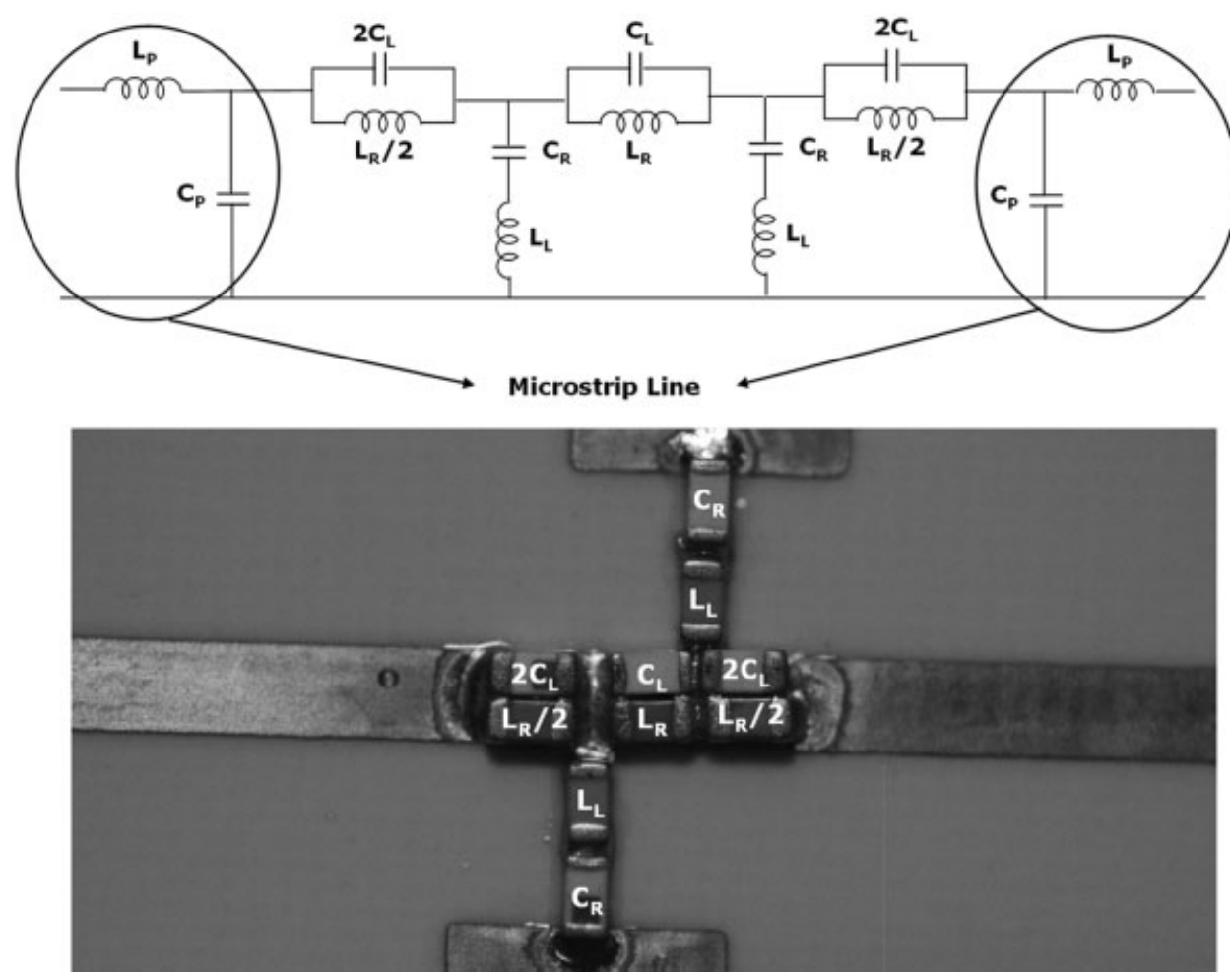

Figure 3 Schematic and photograph of the DL TL

arbitrary three frequency bands. In this article, we present fundamental properties of DL TL and possible applications, including their results.

\section{DOUBLE-LORENTZ TRANSMISSION LINE}

Figure 1 represents both the infinitesimal circuit model for a uniform DL $\left(\Delta z / \lambda_{\mathrm{g}} \rightarrow 0\right)$ or the unit cell of an LC network DL TL structure $\left(\Delta z \cdot \lambda_{\mathrm{g}} / 4\right)$. To predict the fundamental property of the DL TL, the following observation was considered. At low frequencies, the dominant components are $L_{\mathrm{R}}, L_{\mathrm{P}}, C_{\mathrm{R}}, C_{\mathrm{P}}$, and the line is therefore RH. In contrast higher the frequency, if both impedance $Z^{\prime}$ and admittance $Y^{\prime}$ have negative values, the structure shows $\mathrm{LH}$ features. Thus, the DL TL exhibits an RH band at low frequencies similar to the D-CRLH and at high frequencies both the LH and RH band exist like CRLH. However, if both $Z^{\prime}$ and $Y^{\prime}$ do not have negative values at any frequency band, $\mathrm{LH}$ characteristic is not presented.

In the so-called balanced case, with the frequency-independent characteristics, the propagation constant and characteristic impedance are given by (1), respectively,

$$
\begin{gathered}
\beta(\omega)=\omega \sqrt{L_{\mathrm{P}}^{\prime} C_{\mathrm{P}}^{\prime}}+\sqrt{\frac{C_{\mathrm{P}}^{\prime}}{L_{\mathrm{P}}^{\prime}}} \frac{\omega L_{\mathrm{R}}^{\prime}}{1-\omega^{2} L_{\mathrm{R}}^{\prime} C_{\mathrm{L}}^{\prime}} \\
Z_{0}=\sqrt{\frac{L_{P}^{\prime}}{C_{P}^{\prime}}}=\sqrt{\frac{L_{\mathrm{L}}^{\prime}}{C_{\mathrm{L}}^{\prime}}}
\end{gathered}
$$

The matching conditions of the termination impedance $Z_{\mathrm{t}}$ and the phase constant of $\beta_{1}, \beta_{2}$, and $\beta_{3}$ at the given triband frequencies $\omega_{1}, \omega_{2}$, and $\omega_{3}$ impose

$$
Z_{0}=Z_{\mathrm{t}}
$$
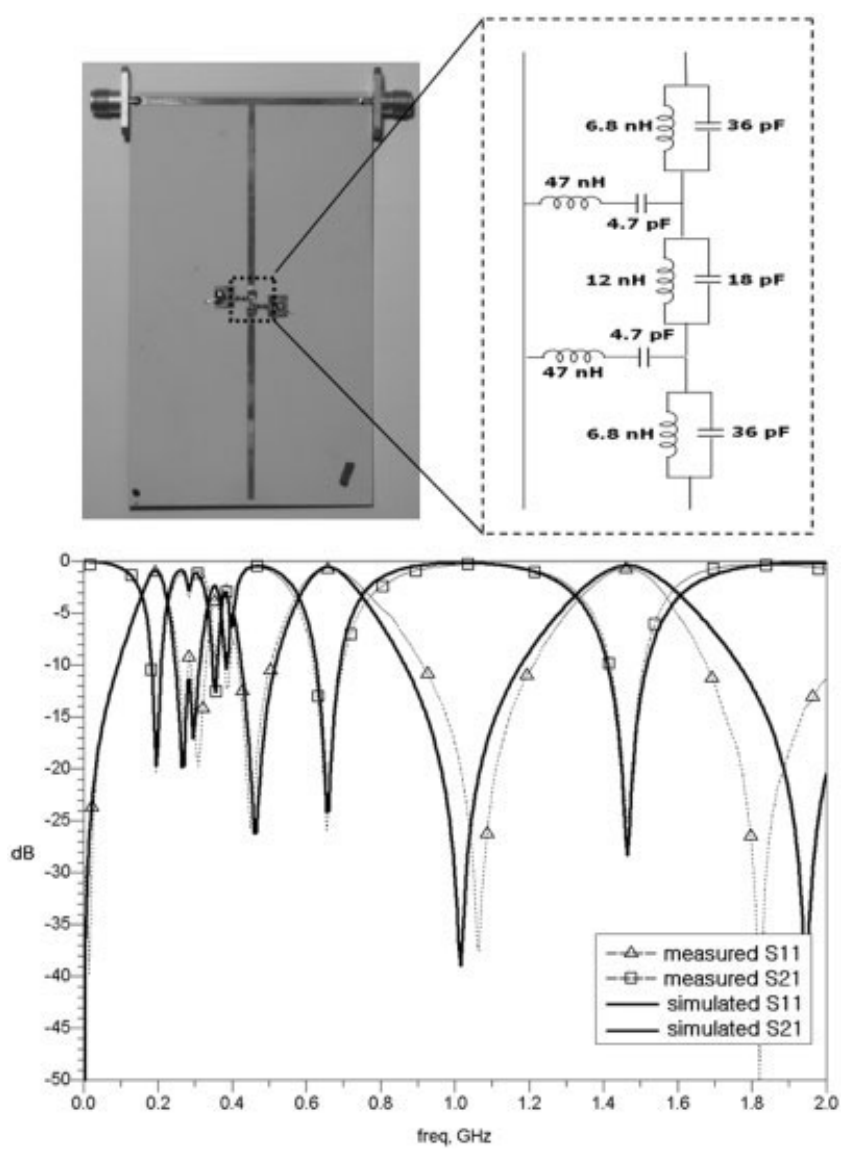

Figure 4 Photograph, schematic, and measured results of the $\lambda / 4 \mathrm{DL}$ open-circuit stub 


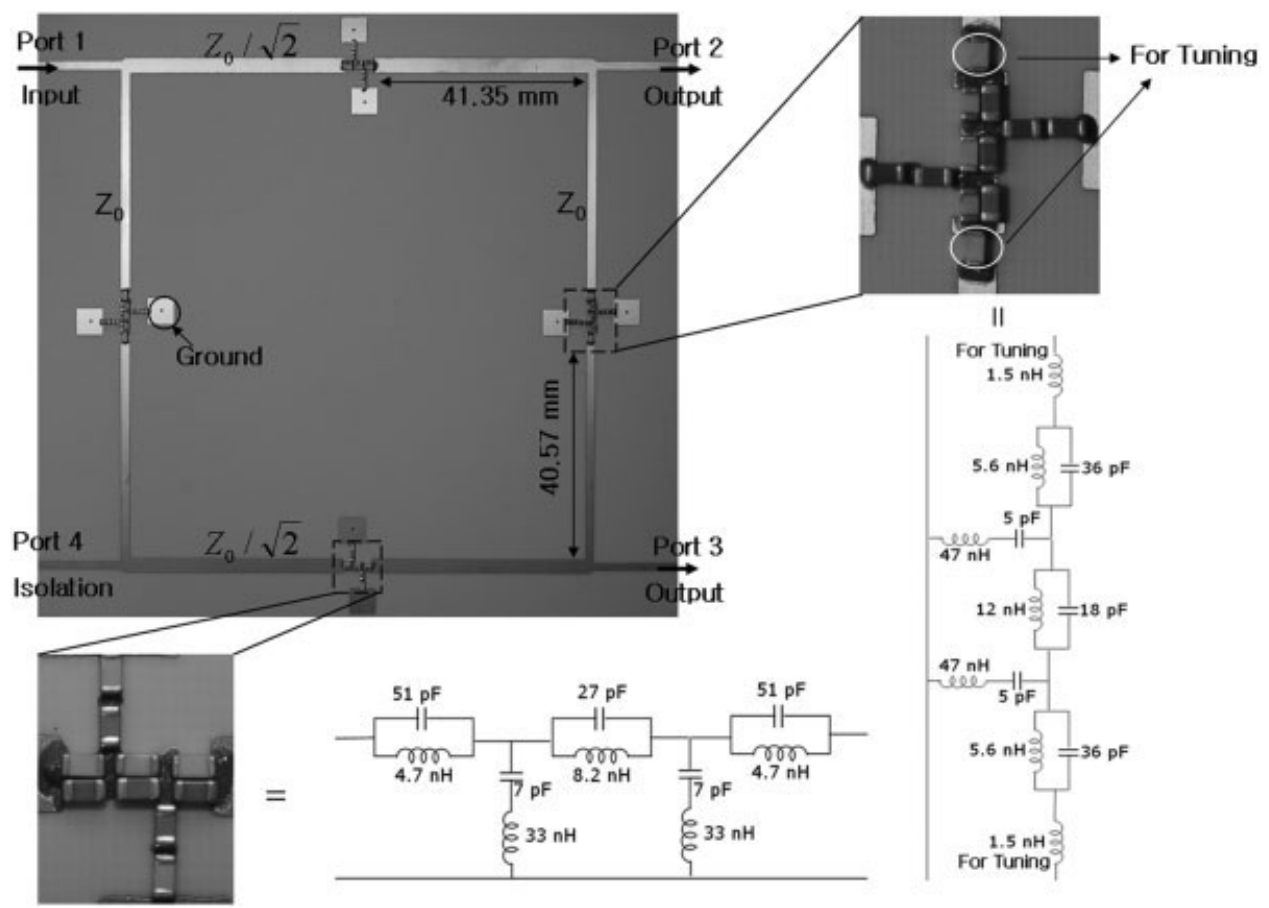

Figure 5 Photograph of the novel BLC and schematics of the fabricated D-CRLH TL sections.

$$
\beta\left(\omega_{1}\right)=\beta_{1}, \quad \beta\left(\omega_{2}\right)=\beta_{2}, \quad \beta\left(\omega_{3}\right)=\beta_{3}
$$

respectively. These equations correspond to five equations because the matching condition ( $2 a$ ) is divided into two equations from (1b). Therefore, there are five equations with the five unknowns $L_{\mathrm{P}}^{\prime}, \quad C_{\mathrm{P}}^{\prime}, \quad L_{\mathrm{L}}^{\prime}, \quad C_{\mathrm{L}}^{\prime}, \quad L_{\mathrm{R}}^{\prime}$ and the triband DL TL circuit parameters depend on $\left(\omega_{1}, \omega_{2}, \omega_{3}\right)-\left(\beta_{1}, \beta_{2}, \beta_{3}\right)$.

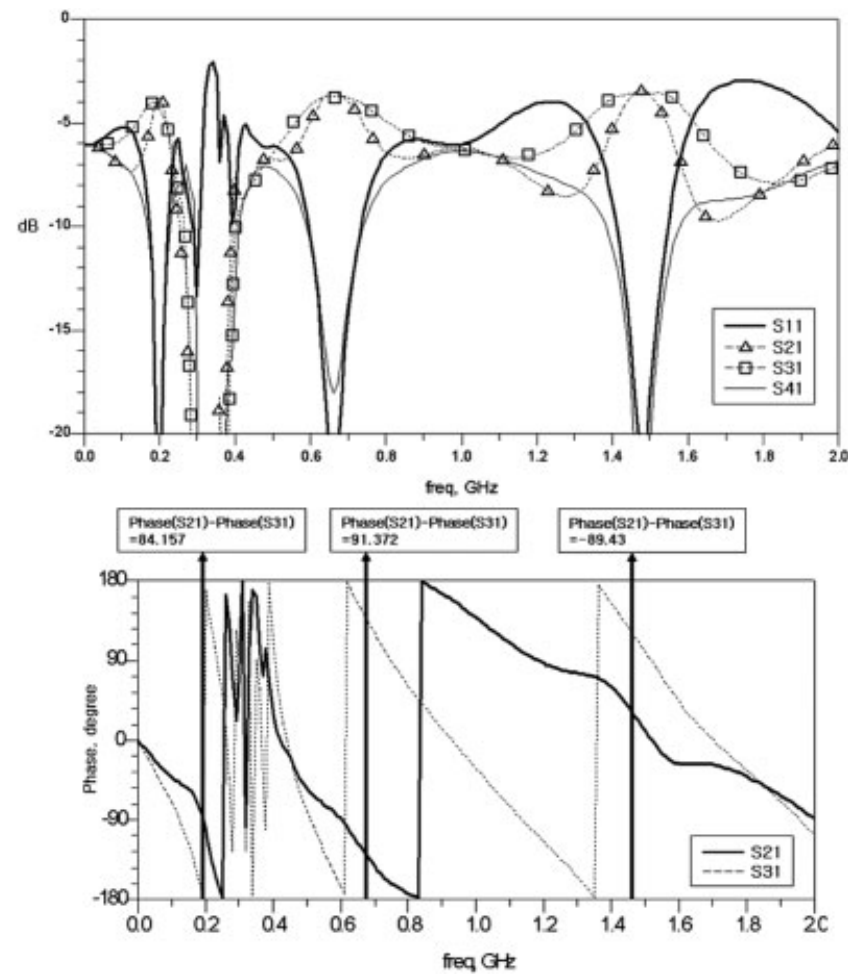

Figure 6 Simulated $S$-parameters of the BLC using DL TLs
Figure 2 shows the characteristics of the DL TL having a triple-band property. Target frequencies of $f_{1}=195 \mathrm{MHz}, f_{2}=$ $670 \mathrm{MHz}$, and $f_{3}=1.465 \mathrm{GHz}$ are selected for mobile TV services [7]. The recent technology trend in this field is to integrate each frequency band operation into one chip. To design the proper DL TL, the DL TL circuit parameters were first determined using (1)

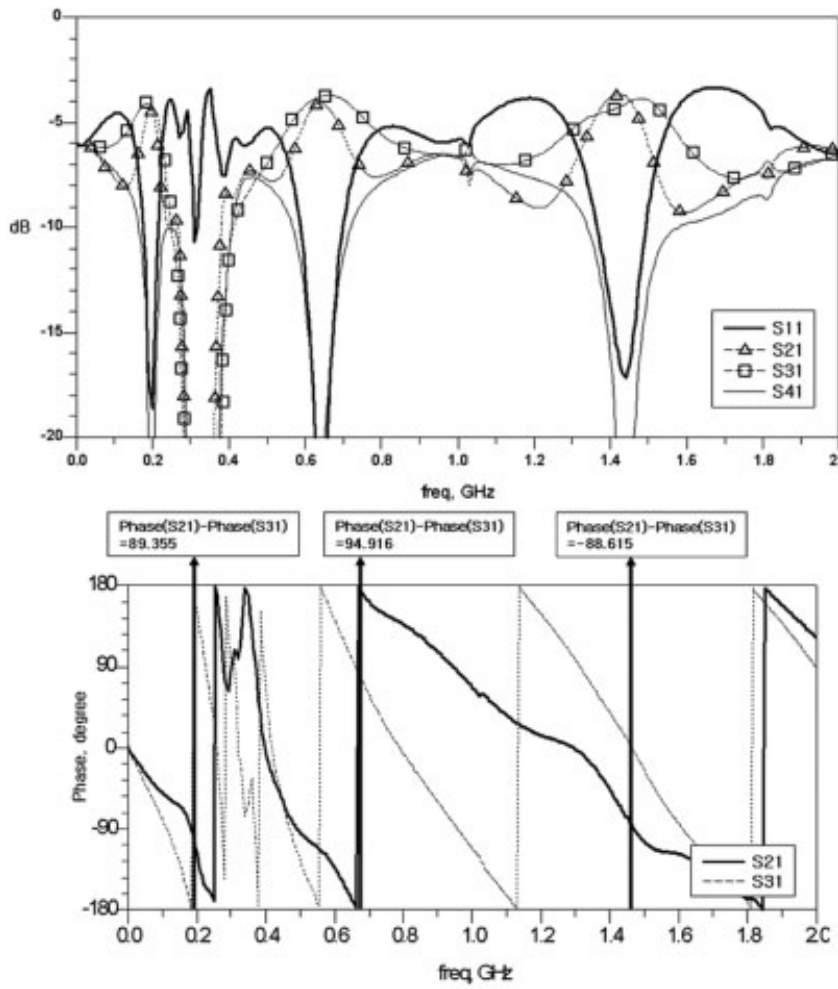

Figure 7 Measured S-parameters of the BLC using DL TLs 
TABLE 1 Performances of the BLC at $195 \mathrm{MHz}$

\begin{tabular}{lll}
\hline & Simulation & Measurement \\
\hline Output 1 $\left(S_{21}\right)$ & $-3.848 \mathrm{~dB}$ & $-4.415 \mathrm{~dB}$ \\
Output 1 $\left(S_{31}\right)$ & $-4.173 \mathrm{~dB}$ & $-4.212 \mathrm{~dB}$ \\
Amplitude imbalance & $0.325 \mathrm{~dB}$ & $0.203 \mathrm{~dB}$ \\
Phase difference & $84.16^{\circ}$ & $89.36^{\circ}$ \\
\hline
\end{tabular}

and (2). The phase response is set to $-\pi / 4, \pi / 4,-\pi / 4$ at $f_{1}, f_{2}$, and $f_{3}$, respectively, for an example of triband DL TL.

\section{TRIBAND $\lambda / 4$ DL TL}

Figure 3 exhibits a schematic and photograph of the DL TL. The D-CRLH section of DL TL consists of two T-type unit cells with parallel resonant structures in impedance part and series resonant structures in admittance part. These resonant structures are implemented by SMT chip components. The RH section of DL TL is accomplished by two microstrip lines on each side of the LH section. In this case, total phase shift $\phi$ is divided into the D-CRLH TL phase shift $\phi^{\mathrm{D}}$ and RH TL phase shift $\phi^{\mathrm{RH}}$. The RH section implemented by microstrip line is designed by determining the characteristic impedance $Z_{\mathrm{cR}}$ and the $\mathrm{RH}$ phase delay $\phi_{1}{ }^{\mathrm{RH}}$, provided in (3a) and (3b), respectively.

$$
\begin{gathered}
Z_{\mathrm{cR}}=\sqrt{\frac{L_{\mathrm{P}}}{C_{\mathrm{P}}}} \\
\phi_{1}^{\mathrm{RH}}=-N \omega_{1} \sqrt{L_{\mathrm{P}} C_{\mathrm{P}}}
\end{gathered}
$$

In the above equation, $N$ is the number of unit cells.

Figure 4 shows a triband $\lambda / 4 \mathrm{DL}$ open-circuit stub at the frequencies $f_{1}=195 \mathrm{MHz}, f_{2}=670 \mathrm{MHz}$, and $f_{3}=1.465 \mathrm{GHz}$. The open stub on a transmission line exhibits the characteristic $\mathrm{S}_{11} \cong 0 \mathrm{~dB}$ at $f_{1}, f_{2}$, and $f_{3}$. The measured results are well agreed with simulated results. All SMT chip components used in implementation of DL TL are provided by the KORCHIP Corporation and Murata Manufacturing Company and their size is $1.6 \mathrm{~mm} \times$ $0.8 \mathrm{~mm}$. In this article, all circuits are fabricated on RO4350 substrates with a dielectric constant of 3.48 and a thickness of $0.762 \mathrm{~mm}$

\section{TRIBAND BRANCH LINE COUPLER}

A triband BLC is fabricated using $\lambda / 4$ DL TLs designed at target frequencies $f_{1}, f_{2}$, and $f_{3}$ instead of $\lambda / 4$ RH TLs used in a conventional BLC. To obtain a triband BLC, the phase responses of the DL TLs are $-90^{\circ},-90^{\circ}$, and $-270^{\circ}$ at $f_{1}, f_{2}$, and $f_{3}$, respectively. At $f_{1}$ and $f_{2}$, the triband BLC operates in the same way as the conventional BLC. At $f_{3}$, the triband BLC works in the very similar way as the former case. The only difference between two cases is the sign of the phase difference between the output signals.

Figure 5 shows the photograph of the implemented triband BLC circuit and its size is $89.6 \mathrm{~mm} \times 93.1 \mathrm{~mm}$. Target frequencies of $f_{1}=195 \mathrm{MHz}, f_{2}=670 \mathrm{MHz}$, and $f_{3}=1.465 \mathrm{GHz}$ are selected for mobile TV services. The first frequency is terrestrial digital

TABLE 2 Performances of the BLC in $670 \mathrm{MHz}$

\begin{tabular}{lll}
\hline & Simulation & Measurement \\
\hline Output 1 $\left(S_{21}\right)$ & $-3.548 \mathrm{~dB}$ & $-4.415 \mathrm{~dB}$ \\
Output 1 $\left(S_{31}\right)$ & $-3.718 \mathrm{~dB}$ & $-4.212 \mathrm{~dB}$ \\
Amplitude imbalance & $0.17 \mathrm{~dB}$ & $0.203 \mathrm{~dB}$ \\
Phase difference & $91.37^{\circ}$ & $94.92^{\circ}$ \\
\hline
\end{tabular}

TABLE 3 Performances of the BLC in $1.465 \mathrm{GHz}$

\begin{tabular}{lll}
\hline & Simulation & Measurement \\
\hline Output 1 $\left(S_{21}\right)$ & $-3.508 \mathrm{~dB}$ & $-4.420 \mathrm{~dB}$ \\
Output 1 $\left(S_{31}\right)$ & $-3.642 \mathrm{~dB}$ & $-3.903 \mathrm{~dB}$ \\
Amplitude imbalance & $0.134 \mathrm{~dB}$ & $0.517 \mathrm{~dB}$ \\
Phase difference & $-89.43^{\circ}$ & $-88.615^{\circ}$ \\
\hline
\end{tabular}

multimedia broadcasting (TDMB) band three frequency band, the second frequency is digital video broadcasting-handheld (DVB-H) frequency band, and the third frequency is TDMB L-band frequency band [7]. Notice that additional inductors are added to the $Z_{0}$ DL TLs due to two factors: the availability of SMT chip components is available only at the discrete values and there is some variation on the reactance and suspectance of the chip components. These effects induce variations in the characteristic impedance of the TLs that result in an imbalance between two output signals. To compensate for these problems, tuning inductors are accompanied and are considered in the simulation.

Figures 6 and 7 exhibit the simulated and measured results of the triband BLC, respectively. The results in all passbands are summarized in Tables $1-3$. In both simulation and measurement, the amplitude imbalance between output signals is less than $1 \mathrm{~dB}$ at $f_{1}, f_{2}$, and $f_{3}$. The triband BLC also has insertion loss of smaller than $4.569 \mathrm{~dB}$, return losses larger than $14 \mathrm{~dB}$, isolations larger than $15 \mathrm{~dB}$, phase differences of output $90^{\circ} \pm 5^{\circ}$ at each target band.

\section{CONCLUSION}

Novel triband microwave components using $\lambda / 4$ DL TLs have been presented. The fundamental theory and implementation of these devices also have been showed. An arbitrary triband operation is accomplished by using the flexible phase slope characteristic of the DL TL with the frequency. The $\lambda / 4$ DL shunt opencircuit stub was shown to block signal at three arbitrary frequencies. A triband BLC was also fabricated using $\lambda / 4$ DL TLs. The DL TL can be used in many microwave applications requiring multiband operation.

\section{ACKNOWLEDGMENTS}

This work was supported by the IT R\&D program of MC/IITA.

\section{REFERENCES}

1. C. Caloz and T. Itoh, Electromagnetic metamaterials, transmission line theory and microwave application, Wiley, New York, 2005.

2. G.V. Eleftheriades, A.K. Iyer, and P.C. Kremer, Planar negative refractive index media using periodically L-C loaded transmission line, IEEE Trans Microwave Theory Tech 50 (2002), 2702-2712.

3. I. Lin, M. Vincentis, C. Caloz, and T. Itoh, Arbitrary dual-band components using composite right/left-handed transmission lines, IEEE Trans Microwave Theory Tech 50 (2004), 1142-1149.

4. C. Caloz, Dual composite right/left-handed (D-CRLH) transmission line metamaterial, IEEE Microwave Wireless Compon Lett 16 (2006), 585-587.

5. A. Rennings, T. Liebig, C. Caloz, and I. Wolff, Double-Lorentz transmission line metamaterial and its application to tri-band devices, IEEE MTT-S Int Microwave Symp, Honolulu, HI (2007), 1427-1430.

6. D.M. Pozar, Microwave engineering, Wiley, New York, 1998.

7. A. Kumar, Mobile TV, DVB-H, DMB, G3 systems and rich media applications, Butterworth-Heinemann, 2007.

(C) 2008 Wiley Periodicals, Inc. 